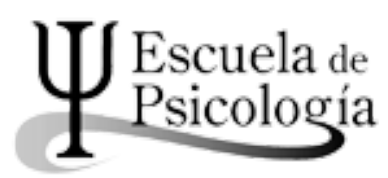

Wímb lu, Rev. electrónica de estudiantes Esc. de psicología, Univ. de Costa Rica. 11 (1): 39-57, 2016 / ISSN: 1659-2107

\title{
Comprensión lectora, variables cognitivas y prácticas de lectura en escolares cubanos
}

\author{
Reading comprehension, cognitive variables and reading practices in Cuban school children
}

\author{
Klency González Hernández ${ }^{a}$ \\ Laura Arango Rodríguez ${ }^{b}$ \\ Neisbet Blasco Fanegoc \\ Kesia Quintana Arteaga ${ }^{d}$
}

\begin{abstract}
Resumen: La investigación pretende determinar la contribución de variables cognitivas (cociente intelectual, fluidez, vocabulario) y prácticas de lectura, al desempeño en comprensión lectora en escolares cubanos de $6^{\text {to }}$ grado. Participaron 72 estudiantes, 34 niñas y 38 varones, con edad promedio de 11 años y residentes de los municipios Plaza de la Revolución, Diez de Octubre y Guanabacoa. Se emplea una metodología mixta. Se aplicaron 4 test de forma individual para evaluar cociente intelectual, fluidez, vocabulario y comprensión. Se utilizaron la entrevista y observación para obtener información sobre las prácticas de lectura. Los resultados muestran que la 57 estudiantes (para un $79 \%$ ) no presentan dificultades de comprensión. El cociente intelectual, la fluidez lectora y el vocabulario tienen un impacto en la comprensión de textos, lo que significa que un desempeño elevado en estas variables tributa a un mejor rendimiento lectocomprensivo. Las prácticas de lectura de los estudiantes también impactan la forma de comprender los textos.
\end{abstract}

Palabras Clave: comprensión lectora, cociente intelectual, fluidez lectora, vocabulario, prácticas de lectura.

a Universidad de la Habana, Facultad de Psicología. Profesora e Investigadora. Máster en Psicología Educativa. Cuba. Correo electrónico: klency@psico.uh.cu

b Universidad de la Habana, Facultad de Psicología. Estudiante sexto año. Cuba. Correo electrónico: larango@estudiantes.psico.uh.cu

c Universidad de la Habana, Facultad de Psicología. Estudiante sexto año. Cuba Correo electrónico: nblasco@estudiantes.psico.uh.cu

d Universidad de la Habana, Facultad de Psicología. Estudiante sexto año. Cuba Correo electrónico: kquintana@estudiantes.psico.uh.cu

Recepción: 5/6/2015 Aceptación: 14/10/2015. 
Abstract: The investigation seeks to determine the contribution of some variable cognitivas (intellectual quotient, reading fluency, vocabulary) and the reading practices, to the acting participated, with age 11 year-old average and residents of the municipalities Plaza de la Revolución Diez de Octubre and Guanabacoa. A mixed methodology is used. 4 test in an individual way was applied to evaluate intellectual quotient, fluency, vocabulary and comprehension. The interview and observation were used to obtain information on the reading practices. The results show that most of the students (79\%) they don't present difficulties of understanding. The intellectual quotient, the reader fluency and the vocabulary have an impact in text's comprehension, what means that high performance in these variables pays to a better reading comprehension. The practices of the students' reading also impact the form of understanding the texts.

Key Words: reading comprehension, cognitive performance, reading fluency, vocabulary, reading practices.

\section{Introducción}

El interés por estudiar la comprensión lectora no es nuevo. Desde principios del siglo $X X$, muchos educadores y psicólogos han considerado la importancia de la lectura y se han ocupado de determinar lo que sucede cuando un lector comprende un texto (Alonso-Tapia, 2005; Dubois, 1991; Duke \& Pearson, 2002). Con el paso del tiempo develar cómo ocurre el proceso de comprensión lectora ha cobrado mayor importancia, por lo que múltiples especialistas han tratado de desarrollar acciones, programas y estrategias de enseñanza-aprendizaje de la lectura. Específicamente los psicólogos cognitivos han dirigido sus investigaciones en dos direcciones. Por un lado, en la búsqueda y análisis de los procesos mentales implicados en la adquisición de la lectura. Por el otro, en definir cómo se utiliza el conocimiento estructurado y preexistente en la memoria y cuáles son los procesos que interactúan para lograr una comprensión del texto (González, 2008).

La comprensión constituye la finalidad del acto de leer y puede ser definida como un proceso interactivo y estratégico que implica la construcción de una representación mental del significado del texto, poniendo en relación las ideas contenidas en el mismo con conocimientos previos almacenados en la Memoria a Largo Plazo (Kintsch \& Van Dijk, 1983; McNamara \& Magliano, 2009; Solé, 1987). Para ello, el lector debe poseer los recursos cognitivos adecuados y emplear estrategias de supervisión, de forma intencional, lo cual se encuentra relacionado con los procesos metacognitivos.

Es durante la educación primaria donde el estudiante debe aprender a comprender. Aproximadamente durante los primeros cuatro años en la escuela se le enseña al niño el código lingüístico, los fonemas y sus reglas de combinación. Una vez aprendido este código el niño está listo para ir más allá de las palabras y aprender a comprender. Sin embargo, en muchas ocasiones no se le enseña explícitamente a desarrollar esta habilidad, ni a usar estrategias para mejorarla.
Estudios a nivel mundial reportan alarmantes índices de prevalencia de las dificultades en comprensión lectora. En el Reino Unido, por ejemplo, aproximadamente un $10 \%$ de los niños entre los 7 y 11 años de edad son malos comprendedores (Ricketts, Nation \& Bishop, 2007; Snowling \& Hulme, 2011). En Estados Unidos, aunque el porcentaje de estudiantes que lee y comprende aumentó de 1997 a 2007, todavía el 33\% de alumnos de cuarto grado y el 26\% de alumnos de octavo grado, continúan leyendo por debajo del nivel básico (Lee, Grigg \& Donahue, 2007). Por su parte, en Argentina e Italia, el porcentaje de estudiantes con dificultades específicas para la comprensión de textos, oscila entre el $5 \%$ y el $10 \%$ de la población escolar total (Abusamra, Ferreres, Raiter, De Beni $\&$ Cornoldi, 2010). En Cuba, investigaciones recientes reportan aproximadamente un $27 \%$ de escolares de quinto grado con dificultades de comprensión (González, Otero \& Castro, 2016).

Muchos son los factores que inciden en las dificultades de comprensión. Existen autores que consideran que las dificultades pueden ser explicadas porque los lectores no usan estrategias apropiadas para comprender el significado de texto y evaluar el proceso (Cain \& Oakhill, 2006; Catts, Adlof \& Ellis 2006; Soriano, Miranda, Soriano, Nievas \& Félix, 2011). Mientras que otros estudios relacionan las dificultades para comprender con otras variables cognitivas -como el cociente intelectual, la fluidez lectora y el vocabulario- (Demagistri, Canet, Naveira \& Richard, 2012; Nation, Cocksey, Taylor \& Bishop, 2010; National Reading Panel; 2000) y procesos de naturaleza metacognitiva -como el conocimiento y control(González, 2011; Peronard, Crespo \& Guerrero, 2001).

La inteligencia es la capacidad mental que comprende funciones como el razonamiento, la planificación, la resolución de problemas, el pensamiento abstracto, la comprensión de ideas complejas y el aprendizaje (Sánchez, García \& González, 2007). Una medida que se obtiene de ella puede ser a través de evaluaciones que den cuenta del cociente intelectual que tengan los estudiantes en un determinado contexto. La relación entre el cociente intelectual y la comprensión no está clara en los estudios consultados. Por una parte, Stuebing, Barth, Molfese, Weiss \& Fletcher (2009) realizaron una exploración bibliográfica de 22 investigaciones que estudiaban la relación de diferentes evaluaciones de cociente intelectual y la respuesta a la intervención de la lectura. Sus resultados revelan que el cociente intelectual no es un fuerte predictor de la respuesta de los sujetos a la intervención de la lectura. Sin embargo, encontraron que para la comprensión lectora, la cantidad promedio de la varianza explicada por el cociente intelectual fue del $15 \%$, lo cual evidencia la relación existente entre el cociente intelectual y la comprensión.

Otra investigación que tiene entre sus objetivos principales explorar la influencia que tenía el cociente intelectual en el rendimiento de la comprensión lectora en un grupo de escolares ingleses, fue realizada por Snowling, Clarke \& 
Nation (2002). Participaron 236 niños de 7 a 9 años de edad, a los que se les aplicó la prueba de inteligencia BAS-II, que utiliza el término "habilidad general conceptual", en vez de inteligencia, para referirse al puntaje obtenido en las habilidades verbales, no verbales y espaciales. Los resultados demostraron que los niños con dificultades en la comprensión mostraban un cociente intelectual ligeramente inferior a la media, y solamente unos pocos tenían un cociente muy bajo. Las conclusiones muestran que el cociente intelectual no resultó tener una relación significativa con la comprensión, y según los autores, esto se debe a que las dificultades de comprensión son específicas, y más bien ligadas a aspectos de procesamiento verbal.

La fluidez se refiere al control que alcanza el lector sobre el nivel superficial del texto, en términos de velocidad, precisión y expresividad, lo cual permite el paso a niveles profundos de procesamiento (Leppanen, Aunola, Niemi, \& Nurmi, 2008). Numerosos autores consideran que la fluidez es un componente necesario -aunque no suficiente- para comprender un texto eficientemente (Sporer, Brunstein \& Kieschke, 2009; Yeo, 2008). Meisinger, Bloom \& Hynd, (2010), investigaron la relación entre fluidez y comprensión lectora en un estudio donde participaron 66 estudiantes de 3er grado y 29 profesores de 25 escuelas primarias de los Estados Unidos. Los profesores debían dividir a los estudiantes en dos grupos distintos: niños que leían las palabras del texto, pero que normalmente no comprendían, y niños que leían con fluidez y entendían lo que leían. Los resultados revelaron que el grupo de los lectores fluidos obtuvo puntuaciones significativamente más altas en los tests de comprensión lectora que el otro grupo. Además, hubo una diferencia significativa en las medidas de lectura fluida entre los dos grupos, siendo el grupo de lectores fluidos el de mayor cantidad de palabras correctas leídas en 1 minuto.

El vocabulario tiene que ver con la amplitud y diversidad léxica que se posee en relación con un idioma determinado. Se refiere a la habilidad para comprender términos y emplearlos para adquirir y transmitir significado (Perfetti, Landi, \& Oakhill, 2005). Al parecer, el vocabulario es uno de los mejores predictores para un desempeño adecuado en comprensión lectora, pues no conocer el uso o sentido de determinadas palabras se convierte en un obstáculo importante para el logro de una representación integrada que dé cuenta de la comprensión (Cromley, Snyder \& Dubois, 2014). Saber los significados de las palabras posibilita crear las conexiones necesarias entre las ideas que provienen del texto y relacionar dichas ideas con la información almacenada en memoria.

Nation, Snowling \& Clark, (2007), estudiaron la relación entre el vocabulario y la comprensión, en una muestra de 83 niños de 8 a 9 años de edad. De esta muestra seleccionaron un grupo de 15 niños con dificultades de comprensión, y 15 sin dificultades. Los resultados reportan que los niños con dificultades de comprensión mostraron un vocabulario más débil que el otro grupo de niños. Los autores explican que el hecho de que el vocabulario esté relacionado con el nivel de comprensión lectora, puede ser argumentado por varias vías. Primero, la extensión del vocabulario de un niño puede limitar la comprensión si el texto que intentan leer contiene palabras que ellos aún no conocen. En segundo lugar, mientras la lectura provee una oportunidad para aprender nuevas palabras, la comprensión puede poner un límite en el desarrollo del vocabulario. Una tercera posibilidad es que la relación entre ambas variables puede estar mediada por procesos compartidos, como por ejemplo el uso del contexto.

Lectura y comprensión tienen también una dimensión social, al realizarse en un contexto socio histórico determinado por las historias de vida de los individuos. Para profundizar en la influencia de lo cultural indagamos en el concepto de prácticas de lectura, que a pesar de no ser unidades de comportamiento observables, implican valores, actitudes, sentimientos y relaciones sociales (Street, 2003). Las prácticas letradas, como procesos internos del individuo, son al mismo tiempo procesos sociales que conectan a las personas entre sí, incluyendo conocimientos compartidos representados en ideologías e identidades sociales. Así, las prácticas toman forma a partir de normas sociales que regulan el uso y la distribución de textos, a la vez que prescriben la posibilidad de producirlos y tener acceso a ellos.

Teniendo en cuenta el concepto de práctica de lectura, las investigaciones deberían indagar en las características que van tomando estas prácticas, los espacios en que ocurren, las historias de lectura de los individuos, el acceso que han tenido a los libros, etc. Al parecer, los lectores exploran diversos espacios acercándose a los libros desde la utilidad que para ellos tienen, rompiendo con los estándares clásicos de dichas prácticas, lo que puede significar que no leen los libros de principio a fin, ni declaran algunas lecturas funcionales por no considerarlas legítimas, aun cuando les hayan aportado conocimientos y vivencias.

La revisión teórica y las investigaciones consultadas demuestran la influencia de múltiples factores sobre la comprensión lectora. Por la importancia que reviste para el desarrollo de programas de intervención en el contexto cubano, así como para contar con mayor cantidad de datos que permitan analizar las dificultades que presentan los estudiantes en relación a este importante proceso, la presente investigación se propone: determinar la contribución de variables cognitivas (cociente intelectual, fluidez y vocabulario) y prácticas de lectura, al desempeño en comprensión lectora en escolares cubanos de $6^{\text {to }}$ grado.

\section{Metodología}

\section{Participantes}

La muestra es no probabilística y está compuesta por 72 estudiantes $(\mathrm{N}=72)$ de sexto grado de La Habana, Cuba. Del total, 34 son niñas (para un 47,2\%) y 38 son varones (para un 52,8\%). La media de edad es de 11,74 años. Estos niños 
se encontraban entre el 25 y el 95 percentil en el Test de Matrices Progresivas Coloreadas de Raven (Raven, Court \& Raven, 1992). Pertenecen a cuatro escuelas primarias de los municipios Plaza de la Revolución, Diez de Octubre y Guanabacoa. La accesibilidad y la territorialidad fueron los elementos definitorios para escoger los municipios.

\section{Instrumentos}

Este es un estudio exploratorio y descriptivo que pretende indagar un tema poco estudiado en el contexto cubano; no trata de medir los conceptos que aborda, ni de encontrar las causas del problema (Hernández, Fernández \& Baptista, 2010). A continuación se presenta la descripción de cada uno de los test usados en el estudio.

Test de Matrices Progresivas Coloreadas de Raven (TMPCR): Está constituido por 36 problemas, cada uno de ellos consiste en una figura incompleta que el niño debe completar. Se presentan 6 alternativas de respuestas posibles, donde sólo una es la respuesta correcta y el resto son distractores. La prueba está dividida en 3 series de 12 problemas cada una: A, Ab y B. Constituye una medida tradicional de inteligencia fluida en niños. Por cada respuesta correcta se otorga un punto y se recoge una puntuación total en la ejecución del test. Los puntajes brutos se comparan con una norma de la ejecución esperada para la edad del niño. En este estudio utilizamos la norma de Strauss, Sherman y Spreen (2006), por la cual se asigna un percentil en cinco rangos: Rango I. Intelectualmente superior: percentil 95; Rango II. Intelectualmente Superior al término medio: percentiles 75 y 90; Rango III. Intelectualmente término medio: percentil 50; Rango IV. Inferior al término medio: percentil 25, 10 y 5; Rango $V$ Intelectualmente deficiente: inferior al percentil 5. Los niños que alcanzan un percentil 25 se consideran niños normal bajo; y los que tienen percentil menor o igual a 10, se consideran niños con rendimiento intelectual bajo.

Test Leer para Comprender (TLC) (Ferreres, Abusamra, Casajús, Cartoceti, Squillace \& Sampedro, 2009): Está compuesto por dos textos, narrativo e informativo, con 10 preguntas de opción múltiple cada uno, y cuatro posibles respuestas en cada caso. Los niños deben leer el texto y marcar para cada pregunta la respuesta correcta. Se otorga 1 punto por cada respuesta correcta, para una puntuación máxima de 10 puntos por texto. El test demanda habilidades de construcción de la microestructura del texto, acceso a la macroestructura y conectar la información recibida con conocimientos y experiencias previas. Al finalizar se obtiene un puntaje total que permite determinar el rendimiento lectocomprensivo del estudiante. Los rangos de puntuación para cada uno de los textos son: texto narrativo: Muy débil (0-3), Débil (4-5), Suficiente (6-7) y Óptimo (8-10); texto informativo: Muy débil (0-4), Débil (5-6), Suficiente (7) y Óptimo (8-10) Se utilizan las normas obtenidas para la población cubana (González, Estévez \& Manzano, enviado para publicar).

Test de fluidez lectora (Mosquera, 2011): Se coloca al escolar ante una situación de lectura de un texto previamente seleccionado según su edad. El texto se presentó escrito con letras negras, en Arial 12, sobre un fondo blanco centrado en una hoja de papel. Los niños debían leerlo en voz alta. Se recogió el tiempo de lectura total del texto, la cantidad de errores, y el promedio de palabras leídas por minuto. Se calculó la fluidez lectora a partir de la fórmula: Total de palabras leídas del texto x 60 / tiempo en segundos que tardó el niño en leer. Los textos fueron equiparados en complejidad de contenido, vocabulario y gramática, con e programa Inflesz.1.0. Para esta muestra, se determinó que los estudiantes que logran índices entre 28 a 65 presentan un rendimiento inferior en fluidez; los que alcanzan entre 66-102 obtienen un rendimiento por debajo de la media; índices de 103-139 representan un rendimiento medio; entre 140-176 un rendimiento por encima de la media; e índices entre 177 y 214 apuntan a un desempeño superior. Los niños que obtienen un rendimiento inferior o por debajo de la media tienen dificultades de fluidez. Se compararon estos índices con un grupo control de niños con características similares a los de este estudio.

Test de Vocabulario Oral: Se administra el subtest de vocabulario de WISC-R Intelligence Scale for Children- Revised (Wechsler, 1974). Ese presentan oralmente a los niños una lista de 32 palabras, y ellos deben proveer las definiciones o explicaciones de cada una de ellas. Las respuestas fueron punteadas de acuerdo con sus niveles de precisión y completamiento usando los criterios de clasificación del Manual de Aplicación. Si el niño domina totalmente el significado de la palabra obtendrá una calificación de 2 puntos, si demuestra saber vagamente lo que significa una palabra, su respuesta obtendrá 1 punto, y si no tiene idea de lo que significa la palabra obtendrá 0 puntos. Se determinó para la muestra del estudio que los niños que alcanzan puntuaciones en el rango de 30 a 35 puntos tienen un desempeño inferior en vocabulario; los que tienen puntuaciones en el rango de 36 a 41 tienen un desempeño medio bajo; de 42 a 47 alcanzan un desempeño medio; de 48 a 53 un desempeño por encima de la media, y puntuaciones en el rango de 54 a 60 indica un desarrollo superior del vocabulario. Los niños que obtienen un desempeño inferior o por debajo de la media tienen dificultades de vocabulario. Se compararon estos índices, y se halla media y desviación estándar con un grupo control de niños con características similares a los de este estudio.

Observación: La información sobre el interés por leer, las prácticas de lectura y algunas características de los entornos educativos de los niños que 
pueden incidir en su desempeño en comprensión lectora se recoge a través de 16 observaciones a niños en el aula. Cada observación tuvo una duración de 45 minutos, y fue no participante. Se registran las actividades efectuadas, los textos leídos, la participación de los alumnos, la guía y retroalimentación del docente, además de las dificultades que se presentan. La información se clasifica en categorías y se usa para realizar un análisis cualitativo sobre las entrevistas semiestruturadas: se emplea para profundizar en las prácticas de lectura de algunos estudiantes (padres y maestros lectores o no, frecuencia y calidad de la lectura en el aula, libros en sus casas, uso de las bibliotecas, empleo del tiempo libre, etc.). Se entrevistará el 50\% del grupo de niños con bueno y pobre desempeño en comprensión lectora, más 7 madres, 2 padres y 7 maestras. Las principales categorías a explorar son: entornos lectores, funciones de los libros, objetivos de lectura, accesibilidad, y diversidad.

\section{Procedimiento}

Los estudiantes fueron evaluados -por las autoras del presente estudio- en los meses de febrero-junio del año 2015. Los que alcanzaron en el test de Matrices Progresivas Coloreadas de Raven entre el 25-95 percentil realizaron el test de comprensión de forma colectiva. La evaluación del test de fluidez, y la tarea de vocabulario se realizó después, de forma individual y contrabalanceado a cada niño. Las evaluaciones se realizaron en sesiones de trabajo entre 20 y 25 minutos, en locales de las propias escuelas, con buena iluminación y bajo nivel de ruido.

Se obtuvo permiso para la realización del estudio del Ministerio de Educación. Se solicitó consentimiento informado a todos los directores municipales, a los de cada centro educacional y a los profesores. A los alumnos y a los padres se les preguntó verbalmente su disposición a colaborar en la investigación.

\section{Análisis estadístico}

Para el análisis de los datos se utilizó el software SPSS Statistics v. 20. 0. Fue empleada la estadística descriptiva para hallar medias, frecuencias y desviaciones estándar de las variables cociente intelectual, fluidez, vocabulario, y comprensión lectora. Se utilizó la t de Student para determinar la diferencia de medias de las variables estudiadas entre los estudiantes con y sin dificultades de comprensión. Se realizó un Análisis Correlacional básico (coeficiente de correlación de Pearson), para determinar la relación entre el nivel de comprensión lectora de los escolares y las otras variables estudiadas. Las categorías obtenidas de las entrevistas y las observaciones se usan para realizar un análisis cualitativo sobre las prácticas lectoras y el impacto que tienen sobre la comprensión.

\section{Resultados}

\section{Comprensión lectora y variables cognitivas}

Los resultados obtenidos en la prueba de comprensión lectora se observan en la Tabla 1. De los escolares estudiados, 57 (para un 79,2\%) no presentan dificultades de comprensión, $38(66,7 \%)$ obtienen un rendimiento óptimo y 19 $(33,3 \%)$ obtienen un rendimiento suficiente. Los restantes 15 estudiantes $(20,8 \%)$ si presentan dificultades para comprender los textos, 9 (60\% de este grupo) obtienen un rendimiento débil y el 6 ( $40 \%$ de este grupo) obtienen un rendimiento muy débil. Existen diferencias significativas $(p<0,001)$ entre ambos grupos de estudiantes, los estudiantes que no presentan dificultades en comprensión obtienen puntuaciones superiores a los estudiantes que si presentan dificultades.

TABLA 1.

Resultados de los escolares en la prueba de comprensión lectora.

\begin{tabular}{lllll}
\hline $\mathrm{N}=72$ & $\begin{array}{l}\text { Escolares } \sin \\
\text { dificultades }\end{array}$ & & $\begin{array}{l}\text { Estudiantes con } \\
\text { dificultades }\end{array}$ & \\
\hline & Rendimiento óptimo & $\begin{array}{l}\text { Rendimiento } \\
\text { suficiente }\end{array}$ & Rendimiento débil & $\begin{array}{l}\text { Rendimiento muy } \\
\text { débil }\end{array}$ \\
\hline & 38 & 19 & 9 & 6 \\
\hline Total & 57 & & 15 & \\
\hline$\%$ & 79 & 21 & \\
\hline $\mathrm{p}<0,001$ & & & & \\
\hline
\end{tabular}

Los escolares sin dificultades de comprensión se centran en la tarea y muestran habilidades para extraer el significado del texto, logran controlar voluntariamente la atención y acuden al investigador sólo en caso de no entender alguna pregunta. Ocupan su tiempo releyendo el texto, realizando inferencias y revisando lo que hicieron. Los escolares con dificultades se distraen con facilidad, realizan la prueba por ensayo y error, dejan preguntas en blanco y tienen dificultades para entender la consigna del test.

La relación entre la comprensión lectora y el desempeño cognitivo se muestra en la Tabla 2. De los 57 estudiantes que no presentan dificultades, 24 obtienen un rendimiento inferior al término medio; 15 un rendimiento intelectualmente término medio; 12 un rendimiento superior al término medio y 6 un rendimiento superior. Para este grupo el $57,9 \%$ de los escolares obtiene un rendimiento medio o por encima de la media. En cuanto a los escolares con dificultades encontramos 
que 10 tienen un rendimiento inferior al término medio; 3 un rendimiento medio; y 2 un rendimiento intelectualmente superior a la media, para un 33,3\% de niños con un rendimiento medio o por encima de la media. La correlación entre la comprensión lectora y el desempeño cognitivo fue significativa $(p<0,01)$.

TABLA 2.

Relación entre comprensión lectora y rendimiento cognitivo.

\begin{tabular}{lllll}
\hline & $\begin{array}{l}\text { Intelectualmente } \\
\text { superior }\end{array}$ & $\begin{array}{l}\text { Superior al } \\
\text { término medio }\end{array}$ & $\begin{array}{l}\text { Término } \\
\text { medio }\end{array}$ & $\begin{array}{l}\text { Inferior al } \\
\text { término medio }\end{array}$ \\
\hline $\begin{array}{l}\text { Escolares } \sin \\
\text { dificultades }\end{array}$ & 6 & 12 & 15 & 24 \\
\hline$\%$ & 10,5 & 21,1 & 26,3 & 42,1 \\
\hline $\begin{array}{l}\text { Escolares con } \\
\text { dificultades }\end{array}$ & - & 2 & 3 & 10 \\
\hline$\%$ & - & 13,3 & 20 & 66,7 \\
\hline
\end{tabular}

La relación entre la comprensión lectora y la fluidez se muestra en la Tabla 3. De los estudiantes que no tienen dificultades en comprensión lectora: 6 estudiantes $(10,5 \%)$ alcanzan un desempeño superior, $10(17,5 \%)$ un desempeño medio alto, 22 estudiantes $(38,6 \%)$ tienen un desempeño medio, $11(19,3 \%)$ obtienen un desempeño medio bajo cometiendo errores de cambio de letras fundamentalmente y 8 estudiantes $(14,3 \%)$ presentan dificultades en la fluidez; para un total de 19 estudiantes (33,3\%) con dificultades específicas en fluidez a pesar de no presentar dificultades en la comprensión lectora.

TABLA 3.

Relación entre comprensión lectora y fluidez.

\begin{tabular}{|c|c|c|c|c|c|}
\hline \multirow{2}{*}{$\begin{array}{l}\text { Desempeño } \\
\text { comprensión } \\
\text { lectora }\end{array}$} & \multicolumn{5}{|l|}{$\begin{array}{l}\text { Desempeño en } \\
\text { fluidez }\end{array}$} \\
\hline & $\begin{array}{l}\text { Desempeño } \\
\text { superior }\end{array}$ & $\begin{array}{l}\text { Desempeño } \\
\text { por encima } \\
\text { de la media }\end{array}$ & $\begin{array}{l}\text { Desempeño } \\
\text { medio }\end{array}$ & $\begin{array}{l}\text { Desempeño } \\
\text { por debajo de } \\
\text { la media }\end{array}$ & $\begin{array}{l}\text { Desempeño } \\
\text { inferior }\end{array}$ \\
\hline $\begin{array}{l}\text { Escolares sin } \\
\text { dificultades }\end{array}$ & 6 & 10 & 22 & 11 & 8 \\
\hline$\%$ & 10,5 & 17,5 & 38,6 & 19,3 & 14,3 \\
\hline $\begin{array}{l}\text { Escolares con } \\
\text { dificultades }\end{array}$ & - & 2 & 7 & 2 & 4 \\
\hline$\%$ & - & 13,3 & 46,7 & 13,3 & 26,7 \\
\hline
\end{tabular}

De los estudiantes que sí tienen dificultades en comprensión lectora: 2 $(13,3 \%)$ alcanzan un desempeño por encima de la media en fluidez, $7(46,7 \%)$ un desempeño medio, $2(13,3 \%)$ estudiantes obtienen un desempeño medio bajo y $4(26,7 \%)$ obtienen un desempeño inferior; para un total de 6 estudiantes (40\%) con dificultades en fluidez además de las dificultades detectadas antes en comprensión lectora.

Existe una diferencia significativa $(p<0,001)$ en el desempeño en fluidez en ambos grupos, los estudiantes que no tienen dificultades en comprensión y los que sí tienen dificultades en este proceso. Existe además una correlación significativa entre la fluidez y la comprensión lectora para la muestra total $(N=72)$ y para el grupo de estudiantes que no presentan dificultades en comprensión ( $N=57)$.

La relación entre la comprensión lectora y el vocabulario se muestra en la Tabla 4. De los estudiantes que no tienen dificultades en comprensión lectora: 14 (para un $24,6 \%$ ) obtuvieron un desempeño superior en vocabulario, $10(17,5 \%)$ un desempeño medio alto, $20(35,1 \%)$ un desempeño medio, para un total de 44 estudiantes $(77,2 \%)$ que tienen un vocabulario extenso y/o acorde a su edad cronológica. Los otros 13 estudiantes $(22,8 \%)$ de este grupo alcanzan un rendimiento por debajo de la media lo que indica dificultades en el vocabulario.

De los estudiantes que sí tienen dificultades en comprensión lectora: 1 $(6,7 \%)$ obtiene un desempeño superior, $2(13,3 \%)$ un desempeño por encima de la media y $2(13,3)$ un desempeño medio, para un total de 5 estudiantes $(33,3 \%)$ que no tienen dificultades en vocabulario. Los 10 estudiantes restantes $(66,7 \%)$ : $4(26,7 \%)$ obtienen un desempeño medio bajo y $6(40 \%)$ obtienen un desempeño inferior, lo que indica en ambos casos dificultades en el vocabulario que impactan la comprensión lectora.

TABLA 4.

Relación entre comprensión lectora y vocabulario.

\begin{tabular}{|c|c|c|c|c|c|}
\hline \multirow{2}{*}{$\begin{array}{l}\text { Desempeño } \\
\text { comprensión } \\
\text { lectora }\end{array}$} & \multicolumn{5}{|l|}{$\begin{array}{l}\text { Desempeño en } \\
\text { vocabulario }\end{array}$} \\
\hline & $\begin{array}{l}\text { Desempeño } \\
\text { superior }\end{array}$ & $\begin{array}{l}\text { Desempeño } \\
\text { por encima } \\
\text { de la media }\end{array}$ & $\begin{array}{l}\text { Desempeño } \\
\text { medio }\end{array}$ & $\begin{array}{l}\text { Desempeño } \\
\text { por debajo de } \\
\text { la media }\end{array}$ & $\begin{array}{l}\text { Desempeño } \\
\text { inferior }\end{array}$ \\
\hline $\begin{array}{l}\text { Escolares sin } \\
\text { dificultades }\end{array}$ & 14 & 10 & 20 & 13 & - \\
\hline$\%$ & 24,6 & 17,5 & 35,1 & 22,8 & - \\
\hline $\begin{array}{l}\text { Escolares con } \\
\text { dificultades }\end{array}$ & 1 & 2 & 2 & 4 & 6 \\
\hline$\%$ & 6,7 & 13,3 & 13,3 & 26,7 & 40 \\
\hline
\end{tabular}


Existe una diferencia significativa $(p<0,001)$ en el desempeño en vocabulario en ambos grupos, los estudiantes que no tienen dificultades en comprensión y los que sí tienen dificultades en este proceso. Existe además una correlación significativa entre el vocabulario y la comprensión lectora para la muestra total $(N=72)$ y para ambos grupos: los estudiantes in dificultades $(N=57)$ y los estudiantes con dificultades $(\mathrm{N}=15)$ en comprensión lectora.

\section{Comprensión y prácticas de lectura}

En el grupo de niños que no tienen dificultades de comprensión, hay 29 $(50,9 \%)$ que expresan estar motivados por la lectura. De ellos, $5(17,3 \%)$ refieren leer diariamente; 20 (69\%) manifiestan leer con frecuencia; y $4(13,7 \%)$ señalan leer a veces. De acuerdo a los escenarios en los cuales practican la lectura, los resultados reflejan que la escuela ocupa el lugar más importante, señalado por 11 escolares (37,9\%), le sigue la casa, escogida por 10 alumnos (para un 34,5\%) y luego la opción "en otro lugar", elegida por 8 alumnos (27,6\%) y referida al trabajo de las madres. Los géneros preferidos por estos estudiantes son: novelas $(83,4 \%)$, revistas $(79 \%)$, letras de canciones $(65,7 \%)$ y episodios de una serie animada llamada Pubertad (25,3\%), que trata temas actuales y conflictos de los adolescentes. Los temas preferidos son: aventuras $(86,7 \%)$, historia $(77,41 \%)$, biología $(70,96 \%)$ y música $(61,29 \%)$, aunque hay 11 estudiantes $(37,9 \%)$ que necesitan niveles de ayuda para identificar estos temas. Los restantes 28 niños de este grupo (lo que representa el $49,1 \%$ ) expresan que no les gusta leer.

De los estudiantes que sí tienen dificultades en comprensión lectora, hay $8(53,3 \%)$ que manifiestan estar motivados por la lectura, $6(75 \%)$ dicen que les gusta leer con frecuencia y $2(25 \%)$ que les gusta leer a veces. En cuanto a los escenarios en los que practican la lectura aparece la escuela como el lugar más importante (5 niños para un $62,5 \%$ ), seguido de la opción "otro lugar" (3 niños, 37,5\%) que se refiere a la casa donde hacen las tareas escolares bajo la supervisión generalmente de un maestro jubilado del sistema de educación cubano, que se dedica a dar orientación a niños en horarios extracurriculares. Los géneros preferidos por estos estudiantes son: historietas $(77,3 \%)$ y letras de canciones $(64,9 \%)$, aunque hay 4 estudiantes $(50 \%)$ que necesitan ayuda para identificar estos géneros. Los temas preferidos son: aventuras $(73,8 \%)$, ciencia ficción $(64,7 \%)$ y música $(59,29 \%)$. Los restante 7 estudiantes $(46,7 \%)$ expresan directamente que no les gusta leer.

No existen diferencias significativas entre las lecturas declaradas por los escolares de ambos grupos (sin dificultades en comprensión, con dificultades en comprensión). Tampoco se consideran las bibliotecas escolares como fuentes de información y obtención de libros, sólo 6 niños $(10,5 \%)$ del grupo de estudiantes sin dificultades y $1(6,7 \%)$ de los estudiantes con dificultades manifiestan visitar este espacio.

En cuanto a las maestras se obtiene que 5 (para un $71,4 \%$ ) ponderan la práctica de la lectura como muy importante pero al referirse a la forma concreta en que la ejercen no dan evidencias de que la lectura ocupe un lugar importante en sus vidas o sea esencial para preparar los materiales escolares. Sólo 2 profesoras de Lengua-Española legitiman la lectura como un medio que les permite prepararse mejor en su rol, además de entretenerse. Algo similar ocurre con las madres, 7 del grupo de niños sin dificultades de comprensión afirman ser lectoras activas pero no aparecen indicadores que demuestren esa afirmación. Las madres del grupo de niños con dificultades tienen respuestas estereotipadas y poco elaboradas en relación con sus prácticas de lectura. permitan darle a la lectura un lugar esencial en su vida cotidiana. En cuanto a los padres se obtiene que mencionan la lectura como una opción recreativa de tiempos pasados.

\section{Discusión}

El propósito general del estudio fue determinar la contribución de variables cognitivas (cociente intelectual, fluidez, vocabulario) y prácticas de lectura al desempeño en comprensión lectora en escolares cubanos de $6^{\text {to }}$ grado de La Habana, Cuba. Los resultados encontrados muestran una influencia positiva significativa del cociente intelectual, la fluidez lectora, el vocabulario y las prácticas de lectura sobre la comprensión lectora; lo que se corresponde con resultados obtenidos anteriormente (Chartier, 2004; Hong y Diamond, 2012; Mosquera, 2011; Peroni, 2003).

En relación a los efectos obtenidos en la aplicación del test de comprensión se obtiene que la prevalencia de las dificultades de comprensión es un poco mayor que la encontrada en niños de la misma edad en países como Inglaterra, Italia, Argentina y EEUU (Carretti, Borella, Cornoldi, \& De Beni, 2009; Rickets et al., 2007). Incluso es mayor que la encontrada en Cuba por González et al. 2016, donde los escolares que tienen dificultades representan el $16 \%$ del total. Estos datos merecen un seguimiento y han de constituir una señal de alerta para educadores e investigadores cubanos.

Los análisis posteriores donde se relaciona la comprensión lectora con otras variables cognitivas arrojan que los estudiantes sin dificultades en comprensión tienen características similares a las halladas por McNamara \& Magliano (2009), quienes plantean que los buenos lectores leen basados en una meta de lectura, ajustándose a un estilo enfocado a esta meta, tienen mayor conciencia de las estrategias y son capaces de saber cuándo es más apropiado utilizarlas. Más de la mitad de estos estudiantes $(66,6 \%)$ obtienen buenos resultados en la 
tarea de fluidez lectora, leyendo de forma rápida, sin cometer errores y con una expresividad adecuada (Soriano et al., 2011); sin embargo, el resto $(33,4 \%)$ tienen dificultades específicas de fluidez lectora aunque estas dificultades no impactan la forma en que comprenden los textos que leen (Snowling \& Hulme, 2011). Sería interesante en investigaciones futuras estudiar nuevamente este grupo de niños desde una perspectiva interventiva.

El grupo de estudiantes con dificultades de comprensión cometen como promedio 16 errores en la prueba para evaluar este proceso cognitivo, mostrando dificultades para la realización de inferencias, lo que les provoca, muchas veces, lagunas en la comprensión (McNamara \& Magliano, 2009). El 40\% de estos estudiantes también tienen dificultades de fluidez lo que impacta sus procesos de comprensión, ya que estos niños no con capaces de decodificar las ideas expresadas en el texto y entender el significado de cada palabra, lo que trae como consecuencia mayores dificultades para elaborar macroideas y realizar inferencias (Sporer et al., 2009).

Las diferencias significativas encontradas entre la fluidez y la comprensión lectora, así como la correlación entre ambos procesos corrobora lo planteado en la literatura en tanto la lectura fluida es aquella que se realiza sin esfuerzo y de forma automática, permitiendo a los niños que así leen dedicar la mayor parte de sus recursos atencionales a la construcción del significado de lo que se lee (Meisinger et al., 2010; National Reading Panel, 2000). La no correlación entre la fluidez y la comprensión en el grupo de estudiantes con dificultades de compresión puede explicarse por el número de sujetos que se ubican en este grupo $(\mathrm{N}=15)$. A pesar de este dato, los resultados obtenidos son suficientes para poder afirmar que en esta muestra de estudio las dificultades en relación a la fluidez lectora influyen también en las dificultades de comprensión.

La relación entre vocabulario y comprensión lectora muestra que los estudiantes sin dificultades de comprensión obtienen un desempeño más elevado lo que evidencia una mayor amplitud de vocabulario. El $66,7 \%$ de los estudiantes con dificultades en comprensión lectora tienen problemas de vocabulario asociados con un pobre conocimiento de las palabras, lo cual impacta de manera considerable el desarrollo de la habilidad lectora y el aprendizaje (Cain \& Oakhill, 2006; Catts, Adlof, \& Ellis 2006; Nation et al., 2010)

Los análisis posteriores donde se relaciona la comprensión con las prácticas de lectura arrojan que la lectura es vista como una actividad formal y rutinaria, y aunque algunos niños (11 del grupo sin dificultades y 5 del grupo con dificultades en comprensión) practican la lectura en la escuela, la maestra no es una figura que motive hacia la realización exitosa de esta actividad lo que puede traer consecuencias negativas para el aprendizaje y para lograr que los escolares se conviertan en futuros lectores. Conjuntamente, aunque la lectura es valorada positivamente por los escolares esto no quiere decir que ellos la practiquen ni que sea de las actividades preferidas que realicen en su tiempo libro. Al parecer, es una actividad más a desarrollar en el currículo escolar, lo que puede entorpecer que se aproveche al máximo y se disfrute.

Emerge en estos escolares una contradicción entre prácticas reales y prácticas declaradas (Donnat, 2004). Aunque todos los escolares entrevistados comentan estar motivados por la lectura; muchos de ellos cuando tienen que referirse a los últimos títulos leídos, o a sus libros favoritos no son capaces de nombrar ninguno, a no ser lecturas relacionadas con la asignatura Lengua Española o lecturas legitimadas por la escuela como la Edad de Oro. Este resultado nos hace pensar: 1) que los niños no leen a pesar de que declaran que sí lo hacen; 2) que los niños subestiman lo que leen y no lo consideran legítimo para ser declarado. Solamente 4 estudiantes $(14,3 \%)$ del grupo de estudiantes sin dificultades en comprensión lectora identifican títulos de libros distintos a los que se enseñan en la escuela, mostrando dominio de algunos autores, géneros y ventajas que aporta la lectura.

No existen diferencias significativas entre las lecturas declaradas por los escolares de ambos grupos (sin dificultades en comprensión, con dificultades en comprensión). Al parecer la representación social que los niños tienen sobre la lectura se sustenta en considerarla una práctica escolar, por tanto, no usan su tiempo libre para leer. Hipotetizamos que esto puede explicarse: 1) porque las actividades de lectura realizadas en la escuela son rutinarias y casi siempre conllevan a una evaluación donde el niño _sobre todo el que tiene dificultades para comprender_ generalmente no sale bien; además los textos usados a pesar de ser clásicos, están desactualizados y no tienen mucha relación con la vida actual de esos niños. En segundo lugar, porque no tienen en el marco familiar ni escolar mediadores activos que les guste leer y les sugieran temáticas atractivas y acordes a su edad.

Teniendo en cuenta los datos resumidos en este epígrafe podemos afirmar que la variabilidad del desempeño en comprensión de los sujetos de la muestra puede ser explicada por las diferencias de rendimiento en desempeño cognitivo, fluidez lectora y vocabulario. Además, las prácticas de lectura de los escolares impactan la manera en que ellos leen y comprenden los textos. Dificultades de vocabulario, fluidez, unido a prácticas pobres y escenarios de poca lectura generalmente están asociados con estudiantes que tienen dificultades en comprensión.

Asumiendo un concepto amplio de lectura y comprensión, y reconociendo la influencia de las variables cognitivas estudiadas, y de las prácticas de lectura, sería útil potenciar desde estadios iniciales de educación primaria escenarios donde los individuos inicien o mantengan sus prácticas lectoras (Bahloul, 2003). En estos escenarios se deben enseñar explícitamente estrategias de lectura y comprensión que impacten el aprendizaje. Además, deben contar con gran cantidad de libros, diversidad de los temas que traten y facilidad de espacios y horarios para acceder a los mismos (Carrasco, 2008). La lectura 
deberá presentarse en la escuela, la familia, la biblioteca como una actividad placentera y de aprendizaje, para que cada cual se acerque a ella desde sus intereses familiarizándose con visiones y representaciones distintas donde se usen estrategias acorde a los objetivos y metas lectoras, y donde se reconozca y declaren las prácticas funcionales como legítimas.

\section{Bibliografía}

Abusamra, V., Ferreres, A., Raiter, A., De Beni, R. \& Cornoldi, C. (2010). Test Leer para Comprender TLC. Evaluación de la comprensión de textos. Buenos Aires: Paidós.

Alonso Tapia, J. (2005). Claves para la enseñanza de la comprensión lectora. Revista de Educación, número extraordinario, 63-93.

Bahloul, J. (2003). Lecturas precarias. Estudio sociológico sobre los pocos lectores. México: SEP/FCE.

Cain, K. \& Oakhill, J. (2006). Profiles of children with specific reading comprehension difficulties. British Journal of Educational Psychology, 76, 683-696.

Carrasco Altamirano, A. (2008). Entre libros y estudiantes. Guía para promover el uso de las bibliotecas en el aula. México: Paidós.

Chartier, A.M. (2004). La memoria y el olvido, o cómo leen los jóvenes profesores. En B. Lahire, Sociología de la lectura (pp.109-137). España: Gedisa, Colección LEA.

Carretti, B., Borella, E., Cornoldi, C., \& De Beni, R. (2009). Role of working memory in explaining the performance of individuals with specific reading comprehension difficulties: A meta-analysis. Learning and Individual Differences, 19, 246-251.

Catts, Hugh W, Adlof, Suzanne M., \& Ellis Weismer, S. (2006). Language deficits in poor comprehenders: A case for the simple view of reading. Journal of Speech-Language-Hearing Research, 49, 278-293.

Cromley, J., Snyder, L. \& Dubois, U. (2014). Cognitive activities in complex science text and diagrams. Contemporary Educational Psychology, 35 (1), 59-74.

Demagistri, M. S., Canet, L., Naveira, L. \& Richard, M. (2012). Memoria de trabajo, mecanismos inhibitorios y rendimiento lecto- comprensivo en grupos de comprendedores de Secundaria Básica. Revista Chilena de Neuropsicología, $7(2), 72-78$.
Donnat, O. (2004). Encuestas sobre los comportamientos de lectura. Cuestiones de método. En Bernard Lahire (comp.), Sociología de la lectura (pp.59-84). Barcelona: Gedisa.

Dubois. A. (1991). El proceso de la lectura: de la teoría a la práctica. Buenos Aires: Aique.

Duke, N. K. \& Pearson, P. D. (2002). Effective Practices for Developing Reading Comprehension. EEUU: International Reading Association.

Ferguson, L., Bråten, I. \& Strømsø, H. (2012). Epistemic cognition when students read multiple documents containing conflicting scientific evidence: A thinkaloud study. Learning and Instruction, 22, 103-120.

Ferreres, A., Abusamra, V., Casajús, A., Cartoceti, R., Squillace, M. \& Sampedro, B. (2009). Pruebas de screening para la evaluación de comprensión de textos. Neuropsicología Latinoamericana, 1, (1), 41-6.

González, K. (2008). Propuesta de un programa para mejorar la comprensión de textos en estudiantes universitarios. Revista Electrónica Actualidades Investigativas en Educación, 8, (2), 1-31.

González, K. (2011). Una mirada interventiva a los problemas de comprensión de textos: aplicación de un programa". Revista Latinoamericana de Estudios Educativos, XLI, (1-2) 117-135.

González, K., Otero, L., \& Castro, A.M. (2016). Factores asociados a las dificultades de comprensión lectora en escolares cubanos. Actualidades investigativas en educación, 6(1), 1-18.

Hernández, R., Fernández, C. \& Baptista, P. (2010). Metodología de la Investigación. México DF: Mc-Graw Hill.

Hong, S. \& Diamond, K. (2012). Two approaches to teaching young children science concepts, vocabulary, and scientific problem-solving skills. Early Childhood Research Quarterly, 27, 295- 305.

Kintsch, W. \& Van Dijk, T.A. (1983). Toward a model of text comprehension and production. Psychological Review, 88, 363-394.

Lee, J., Grigg, W \& Donahue, P. (2007). The nation's report card: Reading 2007. Washington, D.C.: National Center for Education Statistics, Institute of Education Sciences, Department of Education. 
Leppanen, U., Aunola, K., Niemi, P. \& Nurmi, J. (2008). Letter knowledge predicts Grade 4 reading fluency and reading comprehension. Learning and Instruction, 18, 548-564.

McNamara, D.S. \& Magliano, J. (2009). Towards a Comprehensive Model of Comprehension. En Psychology of Learning and Motivation, pp.297-384, EEUU: Elsevier Inc.

Meisinger, E., Bloom, J. \& Hynd, G. (2010). Reading fluency: implications for the assessment of children with reading disabilities. Ann. of Dyslexia, 60, 1-17.

Mosquera, R. (2011). Valor predictivo del procesamiento de la palabra escrita en la competencia lectora en el idioma español. Un estudio longitudinal. Tesis en opción al título académico de Máster en Neurociencias, La Habana, Centro de Neurociencias de Cuba.

Nation, K., Snowling, M. J. \& Clark, P. (2007). Dissecting the relationship between language skills and learning to read: Semantic and phonological contributions to new vocabulary learning in children with poor reading comprehension. International Journal of Speech-Language Pathology, 9 (2), 131- 139.

Nation, K., Cocksey, J., Taylor, J. S. H. \& Bishop, D. V. M. (2010). A longitudinal investigation of early reading and language skills in children with poor reading comprehension. Journal of Child Psychology \& Psychiatry, 51, 1031- 1039.

National Reading Panel: Teaching Children to Read Reports of the Subgroups (2000). U.S. Department of Health and Human Services Public Health Service National Institutes of Health National Institute of Child Health and Human Development NIH Pub. No. 00-4754. Consultado en 2012. http://www.nichd. nih.gov/publications/nrp/report.cfm

Perfetti, CH., Landi, N. \& Oakhill, J. (2005). The acquisition of reading comprehension skill. En M. Snowling \& CH. Hulme (Eds.), The science of reading: A handbook (227-247). Oxford: Blackwell.

Peronard, M., Crespo, N. \& Guerrero, I. (2001). El conocimiento metacomprensivo en escolares chilenos de Educación Básica. Revista Signos, 49 (34), 149- 164

Peroni, M. (2003). Historias de lectura trayectorias de vida y lectura. México: Fondo de Cultura Económica.

Raven, J. C., Court, J. \& Raven, J. (1992). Standard progressive matrices. Oxford, UK: Oxford Psychologist Press.

Ricketts, J., Nation, K. \& Bishop, D. (2007). Vocabulary is important for some, but not all reading skills. United Kingdom: University of Oxford.
Sánchez, E., García, J. \& González, A. (2007). Can differences in the ability to recognize words cease to have and effect under certain reading conditions. Journal of Learning Disabilities, 40, 290-305.

Snowling, M, Clarke, P \& Nation, K. (2002). General cognitive ability in children with Reading comprehension difficulties. British Journal of Educational Psychology, 72, 549-560.

Snowling, M.J. \& Hulme, C. (2011). Evidence-based interventions for reading and language difficulties: Creating a virtuous circle. British Journal of Educational Psychology, 81, 1-23.

Solé, I. (1987). Las posibilidades de un modelo teórico para la enseñanza de la comprensión lectora. Infancia y Aprendizaje, 39, 1- 13.

Soriano, M. Miranda, A., Soriano, E. Nievas, F. \& Félix, V. (2011). Examining the Efficacy of an Intervention to Improve Fluency and Reading Comprehension
in Spanish Children with Reading Disabilities. International Journal of Disability, Development and Education, 58(1), 47-59.

Sporer, N., Brunstein, J. \& Kieschke, U. (2009). Improving students' reading comprehension skills: Effects of strategy instruction and reciprocal teaching. Learning and Instruction, 19, 272-286.

Strauss, E.; Sherman, E. \& Spreen, O. (2006). A Compendium of neuropsychological tests: Administration, norms, and commentary. (3rd. ed.). NY: Oxford University Press.

Stuebing, K., Barth, A., Molfese, P., Weiss, B. \& Fletcher, J. (2009). IQ Is Not Strongly Related to Response to Reading Instruction: A Meta-Analytic Interpretation. Except Child, 76, 31-51.

Street, B. (2003). Los nuevos estudios de literacidad. En V. Zavala, M. Niño \& P. Ames, (Eds), Escritura y sociedad. Nuevas perspectivas teóricas y etnográficas (pp.81-107). Lima: Red para el desarrollo de las ciencias sociales en el Perú.

Wechsler, D. (1974). Manual for the Wechsler Intelligence Scale for Children Revised. New York: Psychological Corporation.

Yeo, S. (2008). Relation between 1- Minute CBM Reading Aloud Measure and Reading. United States of America: Minnesota University

\section{(c) (7)}

Esta obra está bajo una licencia de Creative Commons Reconocimiento - NoComercial - CompartirIgual (by-nc-sa) 